\title{
Isovariant mappings of degree 1 and the Gap Hypothesis
}

\author{
REINHARD SCHULTZ
}

\begin{abstract}
Unpublished results of S Straus and W Browder state that two notions of homotopy equivalence for manifolds with smooth group actions_-isovariant and equivariantoften coincide under a condition called the Gap Hypothesis; the proofs use deep results in geometric topology. This paper analyzes the difference between the two types of maps from a homotopy theoretic viewpoint more generally for degree one maps if the manifolds satisfy the Gap Hypothesis, and it gives a more homotopy theoretic proof of the Straus-Browder result.
\end{abstract}

55P91, 57S17; 55R91, 55S15, 55S91

In order to motivate and explain our results more thoroughly, we shall begin with various pieces of background material. Readers who would rather focus on the main results and their proofs may go directly to Section 2.

\section{Background}

Ever since the topological classification of surfaces was discovered, one basic theme in geometric topology has been the reduction of existence and classification questions for manifolds to problems in algebraic topology. A collection of techniques known as surgery theory has been particularly effective in this regard (compare Rothenberg [37, pages 375-378]). For well over four decades topologists have also known that such techniques also have far reaching implications for manifolds with group actions (cf Browder [9] and [37, pages 378-379]). Not surprisingly, many of the most striking applications of surgery theory require some assumption on the manifolds, mappings or structures under consideration, and for group actions the following restriction has been employed quite often:

Standard Gap Hypothesis For each pair of isotropy subgroups $H \supsetneqq K$ and each pair of components $B \subset M^{H}, C \subset M^{K}$ such that $B \varsubsetneqq C$ we have $\operatorname{dim} B+1 \leq \frac{1}{2}(\operatorname{dim} C)$.

A condition of this sort first appeared explicitly in the unpublished Berkeley doctoral dissertation of Sandor H Straus [45], and the importance and usefulness of the restriction became apparent in work of T Petrie [33; 34]; see also Dovermann-Petrie [16], 
Dovermann-Rothenberg [17], and Lück-Madsen [28]. The reason for this is that the Gap Hypothesis automatically implies that certain maps are isovariant (cf Palais [32]): A mapping of $f: X \rightarrow Y$ of spaces with actions of a group $G$ is said to be isovariant if it is equivariant - so that $f(g \cdot x)=g \cdot f(x)$ for all $g \in G$ and $x \in X$ - and for each $x$ the isotropy subgroup $G_{x}$ of all group elements fixing $x$ is equal to the isotropy subgroup $G_{f(x)}$ of the image point (in general one can only say that the first subgroup is contained in the second). Specifically, surgery theory yields equivariant mappings of the form $G / K \times S^{q} \rightarrow G \cdot M^{K}$ where $q \leq \frac{1}{2} \operatorname{dim} M^{K}$, and at one crucial step in the process one uses the fact that such maps are equivariantly homotopic to isovariant maps by general position if the Gap Hypothesis holds. Usually this is expressed in other terms (eg, the image of $\{H\} \times S^{q}$ lies in $G \cdot M^{H}$ and is disjoint from $G \cdot M^{L}$ for all isotropy subgroups properly containing a conjugate of $H$ ), but it is straightforward to verify that the usual version is equivalent to the one stated here in terms of isovariance.

Applications of surgery to group actions that do not require the Gap Hypothesis frequently assume that the underlying maps of manifolds are isovariant or almost isovariant (cf Browder-Quinn [11], the final section of Dula-Schultz [20], the second part of Schultz [43], and Weinberger's book [49]). The general notion of almost isovariance is defined precisely on page 27 of [20], and the most important special case is reproduced below. For the time being, we merely note that

(1) the sets of nonisovariant points $\left(G_{x} \neq G_{f(x)}\right)$ for such a map may be pushed into very small pieces of the domain where they cause no problems,

(2) standard methods of homotopy theory extend directly to a suitably defined category of almost isovariant mappings (cf [20] and Dror Farjoun [19]),

(3) results of [20] show that almost isovariant homotopy and isovariant homotopy are equivalent in many important cases (including all smooth actions of finite cyclic $p$-groups), and a standard conjecture (believed by most workers in the area) states that the same is true for arbitrary smooth actions of finite groups.

In fact, isovariant techniques play a central role in classification results for group actions when the Gap Hypothesis fails; in many cases where such machinery is not used explicitly, the work can readily be interpreted in these terms. A fundamentally important breakthrough in this area was due to Browder and Quinn [11] (see also the commentary on the latter in Hughes-Weinberger [22]), and a more general discussion of the situation in the smooth category - which also extends earlier work on the smooth classification of topologically linear actions on spheres - appears in [43, Section II.1] (see also the final section of [20]). In the piecewise linear and topological categories, there is a distinct body of results which is largely based on techniques 
from controlled topology (eg Weinberger's book [49], the survey articles by HughesWeinberger [22] and Cappell-Weinberger [12], and the doctoral dissertation of A Beshears [8]). A full historical account of the topic is beyond the scope of this paper, but some additional references include the work of A Assadi with Browder [2] and P Vogel [3], the monograph by L Jones on symmetries of disks [25], and the material on symmetries of aspherical manifolds in Weinberger's paper on higher Atiyah-Singer invariants [50].

\section{Focus of this paper}

In this paper we are particularly interested in the following unpublished result, which is due to Straus [45] for actions that are semifree (the group acts freely off the fixed point set) and W Browder [10] more generally. It implies a fairly strong, general and precise connection between almost isovariance and the Gap Hypothesis.

Theorem 1.1 Let $f: M \rightarrow N$ be an equivariant homotopy equivalence of connected, compact, unbounded ( = closed) and oriented smooth $G$-manifolds that satisfy the Gap Hypothesis. Then $f$ is equivariantly homotopic to an almost isovariant homotopy equivalence.

As noted above, in some cases the results of [20] allow one to replace "almost isovariant" by "isovariant" in the conclusion; in particular, this is true if the isotropy subgroups are normal and linearly ordered by inclusion.

Although Theorem 1.1 is a purely homotopy theoretic statement, the proofs in [45] and [10] require fairly deep results from Wall's nonsimply connected surgery theory [47], which in turn depends upon other deep geometric results such as the classification theory for immersions (cf Phillips [35]) and the Whitney process for geometrically eliminating pairs of double points that cancel algebraically (cf Milnor's monograph on the $h$-cobordism theorem [31]). It is natural to ask whether one can prove Theorem 1.1 without relying so extensively on such a large amount of auxiliary material (this is a special case of the classical scientific maxim called Ockham's Razor). In particular, since one can construct a version of obstruction theory for isovariant maps and define obstructions to finding an isovariant deformation of a given equivariant map [20], it is natural to search for a proof that is related to this obstruction theory. More generally, one would also like to understand the obstructions to isovariance for arbitrary equivariant mappings of degree \pm 1 from one smooth manifold to another. Some basic test cases are examples of equivariant degree one mappings mentioned in [10] which are not equivariantly homotopic to isovariant maps; results of K H Dovermann on isovariant normal maps [15] also provide some motivation. 
The main objective of this paper is to analyze the problem of deforming an equivariant degree one map into an isovariant map when the Gap Hypothesis holds, to use this criterion to provide an essentially homotopy-theoretic proof of Theorem 1.1, and to see how the criterion applies to equivariant homotopy equivalences and other basic examples. In contrast to [45] and [10], our approach requires a minimum of input from geometric topology; namely, nonequivariant transversality and standard results on smooth embeddings in the general position range. For the sake of clarity we shall restrict attention to finite group actions that are semifree in the sense described above; if $G$ is cyclic of prime order, then all actions are semifree. We shall also discuss some applications of Theorem 1.1 to cyclic reduced products that were first considered in [45] and a few positive and negative results just outside the range of the Gap Hypothesis (further information on the latter will appear in sequels to this paper).

Acknowledgments I am extremely grateful to Bill Browder for helpful conversations and correspondence regarding his results on the questions treated here, and especially for providing a detailed account of his counterexamples that appear in Section 4 of this paper. I would also like to thank Heiner Dovermann for various conversations involving his work. Comments on the referee for enhancing the exposition of the paper were also valuable and appreciated. The research in this paper was partially supported by National Science Foundation Grants DMS 86-02543, 89-02622 and 91-02711, and also by the Max-Planck-Institut für Mathematik in Bonn, and sources are also gratefully acknowledged.

\section{Statements of main results}

Suppose that $M$ and $N$ are closed, oriented, semifree smooth $G$-manifolds satisfying the Gap Hypothesis such that all components of the fixed point sets, and suppose that $f: M \rightarrow N$ is a $G$-equivariant map of degree 1 . Let $\left\{N_{\alpha}\right\}$ denote the set of components of the fixed point set $N^{G}$, where we may as well assume that $\alpha$ runs through the elements of $\pi_{0}\left(N^{G}\right)$, and suppose that the associated map $f^{G}$ of fixed point sets defines a 1-1 correspondence between the components of $M^{G}$ and $N^{G}$; for each $\alpha$ let

$$
M_{\alpha}=f^{-1}\left(N_{\alpha}\right) \cap M^{G}
$$

and let $f_{\alpha}: M_{\alpha} \rightarrow N_{\alpha}$ denote the partial map of fixed point sets determined by $f$. Denote the equivariant normal bundles of $M_{\alpha}$ and $N_{\alpha}$ in $M$ and $N$ by $\xi_{\alpha}$ and $\omega_{\alpha}$ respectively, and let $S(v)$ generically represent the unit sphere bundle of the vector bundle $v$ (with the associated group action if $v$ is a $G$-vector bundle). 
Theorem 2.1 Suppose we are given the setting above such that $\operatorname{dim} M_{\alpha}=\operatorname{dim} N_{\alpha}$ for each $\alpha$.

(i) If $f$ is homotopic to an isovariant map, then for each $\alpha$ the map $f_{\alpha}$ has degree \pm 1 , and $S\left(\xi_{\alpha}\right)$ is equivariantly fiber homotopy equivalent to $S\left(f^{*} \omega_{\alpha}\right)$.

(ii) If the two conditions in the preceding statement hold, then $f$ is equivariantly homotopic to a map that is isovariant on a neighborhood of the fixed point set.

(iii) If $f$ is isovariant on a neighborhood of the fixed point set, then $f$ is equivariantly homotopic to an isovariant map if and only if $f$ is equivariantly homotopic to a map $f_{1}$ for which the set of nonisovariant points of $f_{1}$ is contained in a tubular neighborhood of $M^{G}$.

Theorem 1.1 will follow immediately from Theorem 2.1 and the specialization of the the latter to equivariant homotopy equivalences.

Theorem 2.2 In the setting of the previous result, suppose that $f$ is an equivariant homotopy equivalence. Then $f$ is equivariantly homotopic to an isovariant homotopy equivalence.

Note If $f$ is an equivariant homotopy equivalence, then some of the assumptions in the setting and statement of Theorem 2.1 are redundant because an equivariant homotopy equivalence determines a 1-1 correspondence of fixed point set components and the dimensions of corresponding components are also equal because the components are homotopy equivalent.

\section{Application to deleted reduced products}

Given a topological space $X$ and an integer $n$, the $n$-fold cyclic reduced product is defined to be the quotient of the product space $X^{n}$ (ie $n$ copies of $X$ ) modulo the action of $\mathbb{Z}_{n}$ on the latter by permuting coordinates, and the deleted cyclic reduced product is the subset of the latter obtained by removing the image of the diagonal $\Delta\left(X^{n}\right)$ consisting of those points whose coordinates are all equal. In his thesis [45] Straus used his version of Theorem 1.1 to obtain the following homotopy invariance property for such spaces:

Theorem 2.3 Let $M$ and $N$ be closed smooth manifolds of dimension $\geq 2$, let $p$ be an odd prime, and suppose that $M$ and $N$ are homotopy equivalent. Let $\mathbb{Z}_{p}$ act smoothly on the $p$-fold self products $\Pi^{p} M$ and $\Pi^{p} N$ (where $\Pi^{p} X=X \times \cdots \times$ $X, p$ factors $)$ by cyclically permuting the coordinates, and let $\mathbf{D}^{p}(M), \mathbf{D}^{p}(N)$ be 
the invariant subsets sets given by removing the diagonals from $\Pi^{p} M$ and $\Pi^{p} N$. Then the deleted reduced cyclic products $\mathbf{D}^{p}(M) / \mathbb{Z}_{p}$ and $\mathbf{D}^{p}(N) / \mathbb{Z}_{p}$ are homotopy equivalent.

As also noted in [45], this result does not extend to compact bounded manifolds, and in fact closed unit disks of different dimensions generate simple counterexamples. The results of Schultz [42] imply that Theorem 2.3 extends to simply connected manifolds if $p=2$, but recent results of R Longoni and P Salvatore [27] imply that the result does not extend to 3-dimensional lens spaces when $p=2$. Further results on the relationship between $\mathbf{D}^{2}(M) / \mathbb{Z}_{2}$ and $\mathbf{D}^{2}(N) / \mathbb{Z}_{2}$ for homotopy equivalent manifolds appear in a paper by P Löffler and R J Milgram [30].

Since the statement and proof of Theorem 2.3 have apparently not appeared previously in print, we shall outline the (fairly straightforward) argument for the sake of completeness.

Sketch of proof We shall first prove the result when $\operatorname{dim} M=\operatorname{dim} N \geq 3$ and then dispose of the remaining cases afterwards. If $f: M \rightarrow N$ is a homotopy equivalence then $\Pi^{p} f: \Pi^{p} M \rightarrow \Pi^{p} N$ is an equivariant homotopy equivalence of closed smooth $\mathbb{Z}_{p}$-manifolds. All actions of $\mathbb{Z}_{p}$ are semifree if $p$ is prime, so this condition holds automatically. Furthermore, the fixed point sets of the action on $\Pi^{p} M$ and $\Pi^{p} N$ are the respective diagonals $\Delta\left(\Pi^{p} M\right) \cong M$ and $\Delta\left(\Pi^{p} N\right) \cong N$, and since

$$
\begin{gathered}
\operatorname{dim} \Delta^{p}\left(\Pi^{p} X\right)=\operatorname{dim} X=\left(\operatorname{dim} \Pi^{p} X\right) / p \leq \\
\frac{1}{3} \operatorname{dim} \Pi^{p} X<\frac{1}{2} \operatorname{dim} \Pi^{p} X-1
\end{gathered}
$$

if $X=M$ or $N$ is at least 3-dimensional and $p$ is odd, then the Gap Hypothesis also holds. Therefore Theorem 1.1 implies that $\Pi^{p} f$ is equivariantly homotopic to an isovariant homotopy equivalence, and the latter in turn yields an equivariant homotopy equivalence from $\mathbf{D}^{p}(M)$ to $\mathbf{D}^{p}(N)$. The induced map of orbit spaces is the desired homotopy equivalence from $\mathbf{D}^{p}(M) / \mathbb{Z}_{p}$ to $\mathbf{D}^{p}(N) / \mathbb{Z}_{p}$.

Suppose now that $\operatorname{dim} M=\operatorname{dim} N \leq 2$. In these cases homotopy equivalent manifolds are homeomorphic, so we can take the homotopy equivalence $f: M \rightarrow N$ to be a homeomorphism. It follows immediately that $\Pi^{p} f$ is a homeomorphism and as such is automatically isovariant. One can now complete the proof as in the last two sentences of the preceding paragraph.

\section{Further results}

The results of [10] also include a uniqueness statement (up to isovariant homotopy) if $M \times[0,1]$ and $N \times[0,1]$ satisfy the Gap Hypothesis. One can also use the methods of 
this paper together with some additional geometric and homotopy theoretic input to prove such a uniqueness result. The necessary machinery to do so will be developed in a subsequent paper.

Since the results of [10] also apply to actions that are not necessarily semifree, it is natural to ask whether the methods of this paper extend. The answer is yes, but a proof would require the introduction of a considerable amount of extra machinery that would take time to develop and might obscure the main ideas, and this is a major reason for sticking with the semifree case. A brief discussion of some tools needed to carry out such extensions appears at the end of Section 3, and we plan to pursue this further in another sequel to this article.

\section{Overview of the paper}

We shall begin Section 3 by proving that the conditions in Theorem 2.1 are necessary for a map $f$ as above to be properly homotopic to an isovariant map. The proof of sufficiency in Theorem 2.1 splits into two steps, which are carried out in Sections 3 and 4. To motivate the first step, observe that an equivariant map of semifree $G$-manifolds is automatically isovariant on the fixed point set, so a natural starting point is to determine whether the given map can be equivariantly deformed to a map that is isovariant on a neighborhood of the fixed point set. If this is possible and we have a map with this additional property, the next step is to determine whether such a map can be further deformed to another one which is isovariant everywhere. Section 5 contains the proofs of Theorems 1.1 and 2.2. Finally, in Section 6 we shall discuss a variety of questions related to the main results. Some concern the interaction between equivariance and isovariance when the Gap Hypothesis holds, while others involve borderline situations in which the Gap Hypothesis inequalities fail, but only by a small amount. There are obvious questions about the extent to which Theorem 1.1 extends to such examples, and answers to such questions turn out to have close connections to issues in equivariant surgery theory in such borderline cases. Sometimes the latter yields generalizations of Theorem 1.1 to situations where the conclusion is not particularly obvious from a homotopy theoretic viewpoint, and in other cases homotopy theory has implications for equivariant surgery theory.

\section{Preliminary adjustments and necessity}

It will be convenient to begin with some notational conventions and elementary observations in order to simplify the main discussion and the proofs. 
Let $P$ be a closed smooth $G$-manifold, where $G$ is a finite group. By local linearity of the action we know that the fixed point set $P^{G}$ is a union of connected smooth submanifolds; as before, denote these connected components by $P_{\alpha}$. For each $\alpha$ let $D\left(P_{\alpha}\right)$ denote a closed tubular neighborhood. By construction these sets are total spaces of closed unit disk bundles over the manifolds $P_{\alpha}$, so let $S\left(P_{\alpha}\right)$ and denote the associated unit sphere bundles; it follows that

$$
\partial\left[D\left(P_{\alpha}\right)\right]=S\left(P_{\alpha}\right) .
$$

Suppose now that $M$ and $N$ are smooth semifree $G$-manifolds and $f: M \rightarrow N$ is an equivariant mapping.

\section{The "only if" direction of Theorem 2.1}

Assume that we have the setting and notation introduced in order to state Theorem 2.1:

(1) $M$ and $N$ are compact, oriented, semifree smooth $G$-manifolds satisfying the Gap Hypothesis.

(2) $f: M \rightarrow N$ is a $G$-equivariant map of degree 1 .

(3) $\left\{N_{\alpha}\right\}$ denotes the set of components of $N^{G}$ where we may as well assume that $\alpha$ runs through the elements of $\pi_{0}\left(N^{G}\right)$.

(4) The associated map $f^{G}$ of fixed point sets defines a 1-1 correspondence between the components of $M^{G}$ and $N^{G}$.

(5) If for each $\alpha$ we let

$$
M_{\alpha}=f^{-1}\left(N_{\alpha}\right) \cap M^{G}
$$

then $f_{\alpha}$ is the continuous map from $M_{\alpha}$ to $N_{\alpha}$ determined by $f$.

(6) If the equivariant normal bundles of $M_{\alpha}$ and $N_{\alpha}$ in $M$ and $N$ are $\xi_{\alpha}$ and $\omega_{\alpha}$ respectively, and let $S(v)$ and $D(v)$ generically represent the unit sphere and disk bundle of the vector bundle $v$ (with the associated group action since $v$ is a $G$-vector bundle).

Not surprisingly, we shall also use the notational conventions we have previously developed and mentioned.

Necessity proof for Theorem 2.1 Each of the first two basic conditions in Theorem 2.1 depends only on the equivariant homotopy class of a mapping of manifolds, so without loss of generality we may replace $f$ by any map in the same proper equivariant homotopy class. In particular, since we are assuming that $f$ is properly equivariantly homotopy to an isovariant map, we might as well assume that $f$ itself is isovariant. 
By the results of [20] (in particular, see Proposition 4.1 on page 27), the map $f$ is isovariantly homotopic to a map $f_{0}$ such that for each $\alpha$ we have $f\left(D\left(M_{\alpha}\right)\right) \subset D\left(N_{\alpha}\right)$, $f\left(S\left(M_{\alpha}\right)\right) \subset S\left(N_{\alpha}\right)$, and

$$
f\left(M-\cup_{\alpha} \text { Int } D\left(M_{\alpha}\right)\right) \subset N-\cup_{\alpha} \text { Int } D\left(N_{\alpha}\right) .
$$

For each choice of $\alpha$ let

$$
h_{\alpha}:\left(D\left(M_{\alpha}\right), S\left(M_{\alpha}\right)\right) \longrightarrow\left(D\left(M_{\alpha}\right), S\left(M_{\alpha}\right)\right)
$$

be the associated map of pairs defined by $f_{0}$. Since the latter has degree 1 , the same is true for each of the maps $h_{\alpha}$. We have already noted that $D(-)$ and $S(-)$ are disk and sphere bundles over the appropriate components of fixed point sets, and therefore a simple spectral sequence argument implies that $(a)$ the degrees of the maps $f_{\alpha}$ are all equal to \pm 1 , up to an equivariant homotopy of pairs the map $h_{\alpha}$ sends a spherical fiber in $S\left(M_{\alpha}\right)$ to a spherical fiber in $S\left(N_{\alpha}\right)$ by a map of degree \pm 1 . Therefore an equivariant analog of a classical result due to A Dold [13] (cf Waner [48]) shows that there is a $g$-equivariant fiber homotopy equivalence from $S\left(\xi_{\alpha}\right)$ to $S\left(f_{\alpha}^{*} \omega_{\alpha}\right)$, where as before $\xi_{\alpha}$ and $\omega_{\alpha}$ denoted the corresponding equivariant normal bundles for $M_{\alpha}$ and $N_{\alpha}$. This completes the proof that Condition (i) holds. Since the set of nonisovariant points for an isovariant map is empty by definition, Condition (iii) is automatically true, so the proof is complete.

\section{Some examples}

It is not difficult to construct equivariant maps of degree 1 which satisfy the Gap Hypothesis but do not satisfy the statements in Condition (i) of Theorem 2.1 on degrees and equivariant normal bundles.

Example 3.1 Let $V$ be a nontrivial semifree real representation of $G$ such that $\operatorname{dim} V^{G}>0$ and the Gap Hypothesis holds, and let $S^{V}$ be the one point compactification, which is equivariantly homeomorphic to the unit sphere in $V \oplus \mathbb{R}$. It is well known that for each positive integer $k$ there is a $G$-equivariant map $h_{k}: S^{V} \rightarrow S^{V}$ such that $\operatorname{deg} h_{k}=1$ and $\operatorname{deg} h_{k}^{G}=k|G|+1$ (eg, this is a very special case of the equivariant Hopf Theorem stated in tom Dieck's book on the Burnside ring and equivariant homotopy theory [46, Theorem 8.4.1, pages 213-214]). Since the fixed point set is connected and the degree of the map on the fixed point set is not \pm 1 if $|G|>2$ or $k \neq 0$, it follows that $h_{k}$ cannot be homotopic to an isovariant map. However, the map $h_{k}$ does satisfy the second part of Condition (i) involving pullbacks of equivariant normal bundles because the equivariant normal bundle of $\left(S^{V}\right)^{G}$ in $S^{V}$ is a product bundle. 
Example 3.2 Let $G$ be a cyclic group, assume that $k, m$ and $r$ satisfy $k \equiv 0(4)$, $k, m>0$ and $2 r>m+k$, and let $\gamma$ be a complex $r$-dimensional vector bundle over $S^{k}$ which represents a generator of $\pi_{k}\left(B U_{r}\right) \cong \mathbb{Z}$. Take $M$ to be the associated $(2 r+m)-$ sphere bundle over $S^{k}$. Then $G$ acts smoothly and fiber preservingly on $M$ with fixed point set $S^{k} \times S^{m}$, and each point has an invariant open neighborhood on which the action of $G$ is smoothly equivalent to the linear representation $V=\mathbb{R}^{k+m} \oplus \mathbb{C}^{r}$. If we collapse everything outside such a neighborhood to a point, we obtain an equivariant map of degree 1 from $M$ to $S^{V}$. This map also has degree 1 on the fixed point sets, but we claim it does not satisfy the pullback condition for equivariant normal bundles. Since the equivariant normal bundle in $M$ is the pullback of $\gamma$ under the coordinate projection map from $S^{k} \times S^{m} \rightarrow S^{m}$, it will suffice to check that $\gamma$ is not equivariantly fiber homotopically trivial. In fact, the underlying nonequivariant vector bundle is well known to be stably fiber homotopically nontrivial (see, for example, Adams [1]).

\section{Normal straightening and relative isovariance obstructions}

In this section we shall prove the implication of Theorem 2.1 in the other direction; namely, if Conditions (ii) is satisfied then one can make $f$ isovariant near the fixed point set, and if (iii) is satisfied then the map $f$ is equivariantly homotopic to an isovariant mapping. The first step is to examine the consequences of Condition (ii).

Proposition 4.1 In the setting of Section 2, suppose that $f: M \rightarrow N$ is a continuous equivariant degree 1 map. Assume that each of the associated maps of fixed point components $f_{\alpha}: M_{\alpha} \rightarrow N_{\alpha}$ has degree \pm 1 and that $S\left(\xi_{\alpha}\right)$ is equivariantly fiber homotopy equivalent to $S\left(f^{*} \omega_{\alpha}\right)$ for each $\alpha$. Then there are closed, pairwise disjoint, equivariant tubular neighborhoods $D\left(M_{\alpha}\right)$ of the fixed point set components $M_{\alpha}$ and an equivariant mapping $f_{0}$ such that $f_{0}$ is equivariantly homotopic to $f$ and for each $\alpha$ the restriction $f \mid D\left(M_{\alpha}\right)$ is isovariant.

Proof For each $\alpha$ choose an equivariant fiber homotopy equivalence $h_{\alpha}: S\left(\xi_{\alpha}\right) \rightarrow$ $S\left(f_{\alpha}^{*} \omega_{\alpha}\right)$, and let $k_{\alpha}$ be the composite of $h_{\alpha}$ with the canonical induced bundle mapping $S\left(f_{\alpha}^{*} \omega_{\alpha}\right) \rightarrow S\left(\omega_{\alpha}\right)$. Define a map $H_{\alpha}: D\left(M_{\alpha}\right) \rightarrow D\left(N_{\alpha}\right)$ using $k_{\alpha}$ and fiberwise radial extension. It follows that $H_{\alpha}$ is equivariantly homotopic to $f \mid D\left(M_{\alpha}\right)$ for each $\alpha$, and hence by the equivariant Homotopy Extension Property we may deform $f$ equivariantly to a map $f_{0}$ such that $f_{0} \mid D\left(M_{\alpha}\right)=H_{\alpha}$ for each $\alpha$. Since each $H_{\alpha}$ is isovariant, it follows that the restriction of $f_{0}$ to a neighborhood of the fixed point set is isovariant as required. 
We shall conclude this section by proving the sufficiency of the condition in (iii). A key step in the proof of Theorem 1.1 will be to prove that an equivariant homotopy equivalence has this property.

Proposition 4.2 Let $M$ and $N$ be as before, and suppose that $f: M \rightarrow N$ is a continuous equivariant map that is isovariant on a neighborhood of the fixed point set. If there is a system of closed tubular neighborhoods $W_{\alpha}$ of $M^{G}$ such that the set of nonisovariant points lies in the interiors of the sets $W_{\alpha}$, then $f$ is equivariantly homotopic to an isovariant map.

Note that we make no assumption about the images of the sets $W_{\alpha}$, and in particular we do not assume that they lie in the tubular neighborhoods of the components of the fixed point set of $N$.

Proof By assumption $f$ is already isovariant on the closed complement of a submanifold $T$ of the form $\cup_{\alpha} S\left(M_{\alpha}\right) \times\left[\frac{1}{2}, 1\right]$ where as usual $S\left(M_{\alpha}\right)$ denotes the boundary of a tubular neighborhood. Let $T_{\alpha}$ be the portion lying over $M_{\alpha}$ and denote the boundary components corresponding to $S\left(M_{\alpha}\right) \times\left\{\frac{1}{2}\right\}$ and $S\left(M_{\alpha} \times\{1\}\right.$ by $\partial_{0} T_{\alpha}$ and $\partial_{1} T_{\alpha}$ respectively. Let

$$
B_{\alpha}=M-\cup_{\beta \neq \alpha} M_{\beta} \quad C_{\alpha}=N-\cup_{\beta \neq \alpha} N_{\beta}
$$

let $S_{\alpha}$ be the spherical fiber of $S\left(M_{\alpha}\right)$, and let $A_{\alpha}$ correspond to the annulus $S_{\alpha} \times\left[\frac{1}{2}, 1\right]$.

For each value of $j$ the map $f$ determines a map of triads

$$
f_{\alpha}:\left(T_{\alpha} ; \partial_{0} T_{\alpha}, \partial_{1} T_{\alpha}\right) \longrightarrow\left(C_{\alpha} ; D\left(N_{\alpha}\right), N-N^{G}\right)
$$

and by the results of Dula-Schultz [20, Section 4] it suffices to show that each such map of triads can be compressed equivariantly rel $\partial_{1} T_{\alpha} \cup A_{\alpha}$ into the triad $\left(N-N^{G} ; S\left(N_{\alpha}\right), N-N^{G}\right)$. The methods of [20, Sections 1 and 5] imply that the obstructions to compression lie in diagram-theoretic Bredon equivariant cohomology groups of the form $B R H^{i}\left(\mathbf{T} ; \Pi_{i}\right)$, where $\mathbf{T}$ is the diagram associated to the triad $\left(T_{\alpha} ; \partial_{0} T_{\alpha}, \partial_{1} T_{\alpha} \cup A_{\alpha}\right)$ and $\Pi_{i}$ is the following diagram of abelian groups:

$$
\pi_{i}\left(D\left(N_{\alpha}\right), S\left(N_{\alpha}\right)\right) \stackrel{\partial_{0}^{\#}}{\longrightarrow} \pi_{i}\left(C_{\alpha}, N-N^{G}\right) \stackrel{\partial_{1}^{\#}}{\longleftarrow}\{0\}
$$

If $q_{\alpha}=\operatorname{dim} M-\operatorname{dim} F_{\alpha}$ then $\left(D\left(N_{\alpha}\right), S\left(N_{\alpha}\right)\right)$ is $\left(q_{\alpha}-1\right)$-connected by the identity $\pi_{s}\left(D\left(N_{\alpha}\right), S\left(N_{\alpha}\right)\right) \cong \pi_{s-1}\left(S_{\alpha}\right)$, and a standard general position argument shows that $\left(C_{\alpha}, N-N^{G}\right)=\left(C_{\alpha}, C_{\alpha}-N_{\alpha}\right)$ is also $\left(q_{\alpha}-1\right)$-connected. Therefore the BlakersMassey Theorem (see Gray [21, page 143]) implies that the map $\partial_{0}^{\#}$ is bijective if $i \leq 2 q_{\alpha}-3$ and surjective if $i=2 q_{\alpha}-2$. In particular, this means that the equivariant 
diagram cohomology groups $B R H^{i}\left(\mathbf{T} ; \Pi_{i}\right)$ reduce to ordinary Bredon cohomology groups ${ }_{B R} H^{i}\left(T_{\alpha}, \partial_{1} T_{\alpha} ; \pi_{i}\left(C_{\alpha}, N-N^{G}\right)\right)$ if $i \leq n-1$ or if $i=n$ and $\operatorname{dim} F_{\alpha}+3 \leq q_{\alpha}$. Since $T_{\alpha} \cong \partial_{1} T_{\alpha} \times[0,1]$ it follows immediately that the relative cohomology groups vanish in all such cases. Since $\operatorname{dim} T_{\alpha}=\operatorname{dim} M=\operatorname{dim} N$, this implies that the isovariance obstructions vanish in all cases except perhaps when $i=n=2 q_{\alpha}-2$. In such cases the value group fits into the following exact sequence, which arises by restricting diagram-theoretic cochains in $C\left(X^{\prime} \rightarrow X ; \pi^{\prime} \rightarrow \pi\right)$ to ordinary cochains in $C\left(X^{\prime} ; \pi^{\prime}\right)$ :

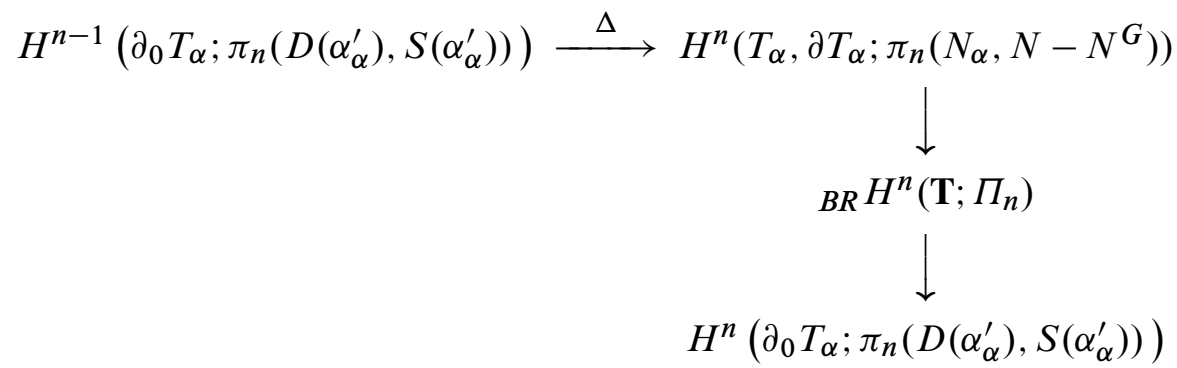

The map $\Delta$ in this sequence is given by combining the coefficient homomorphism for the map $\delta_{0}^{\#}$ in dimension $n$ with the suspension isomorphism

$$
H^{n-1}\left(\partial_{0} T_{\alpha} ; \pi\right) \longrightarrow H^{n}\left(T_{\alpha}, \partial T_{\alpha} ; \pi\right) .
$$

Therefore the Blakers-Massey Theorem, the $(n-1)$-dimensionality of $\partial_{0} T_{\alpha}$, and the Bockstein exact sequence for the short exact sequence

$$
0 \longrightarrow \text { Kernel } \longrightarrow \pi_{n}\left(D\left(\alpha_{\alpha}^{\prime}\right), S\left(\alpha_{\alpha}^{\prime}\right)\right) \stackrel{\partial_{0}^{\#}}{\longrightarrow} \pi_{n}\left(C_{\alpha}, N-N^{G}\right) \longrightarrow 0
$$

imply that $\Delta$ is onto. But the last object in $(\star)$ is zero because $\operatorname{dim} \partial_{0} T_{\alpha}=n-1$, and it follows by exactness that ${ }_{B R} H^{n}\left(\mathbf{T} ; \Pi_{n}\right)$ is also trivial. Therefore the isovariance obstructions always vanish.

The following examples due to Browder [10] show that it is not always possible to deform an equivariant degree 1 map so that it is isovariant near the fixed point set and the set of nonisovariant points lies in a tubular neighborhood of the fixed point set.

Examples 4.3 Let $k$ and $q$ be distinct positive integers such that $q$ is even and $G$ has a free $q$-dimensional linear representation (that is, the group acts freely except at the zero vector). Let $N=S^{k} \times S^{q}$ with trivial action on the first coordinate and the one point compactification of the free linear representation on the second, let $M_{0}$ be the disjoint union of $N$ and two copies of the space $G \times S^{k} \times S^{q}$ (where $G$ acts by translation on itself and trivially on the other two coordinates), and define an equivariant 
map $f_{0}: M_{0} \rightarrow N$ by taking the identity on $M$, the unique equivariant extension of the identity map on $S^{k} \times S^{q}$ over one copy of $G \times S^{k} \times S^{q}$, and the unique equivariant extension of an orientation reversing self diffeomorphism of $S^{k} \times S^{q}$ over the other copy. By construction this map has degree one, and one can attach 1-handles equivariantly to $M_{0}$ away from the fixed point set to obtain an equivariant cobordism of maps from $f_{0}$ to a map $f$ on a connected 1-manifold $M$ that is nonequivariantly diffeomorphic to a connected sum of $2 \cdot|G|+1$ copies of $S^{k} \times S^{q}$. Since the fixed point sets of $M$ and $N$ are $k$-dimensional and the manifolds themselves are $(k+q)$-dimensional, it follows that the Gap Hypothesis holds if we impose the stronger restriction $q \geq k+2$. By construction the map $f$ determines a homotopy equivalence of fixed point sets and is isovariant on a neighborhood of the fixed point set.

Assertion It is not possible to deform $f$ equivariantly so that the set of nonisovariant points lies in a tubular neighborhood of the fixed point set. In particular, it is also not possible to deform $f$ equivariantly to an isovariant map.

Proof To prove the assertion, assume that one has a map $h$ equivariantly homotopic to $f$ with the stated property, and let $U$ be a tubular neighborhood of $M^{G}$ that contains the set of nonisovariant points. Let $X$ be a submanifold of the form $\{g\} \times\{v\} \times S^{q}$ in $M$ that arises from one of the copies of $G \times S^{k} \times S^{q}$ in $M_{0}$. Although $X$ and $U$ may have points in common, by the uniqueness of tubular neighborhoods we can always isotope $X$ into a submanifold $X^{\prime}$ that is disjoint from $U$. By the hypotheses on $h$ we know that $h\left(X^{\prime}\right)$ is disjoint from $N^{G}=S^{k} \times S^{0}$, and therefore $h\left(X^{\prime}\right)$ is contained in

$$
N-N^{G} \cong S^{k} \times S^{q-1} \times \mathbb{R}
$$

so that the image of the generator of $H_{q}\left(X^{\prime}\right)=\mathbb{Z}$ maps trivially into $H_{q}(N)$. However, $h$ is supposed to be homotopic to a map which is nontrivial on the latter by construction, so we have a contradiction, and therefore it is not possible to find an isovariant map $h$ that is equivariantly homotopic to $f$.

A refinement of the preceding argument shows that if $Y$ is a subset of $M$ such that the image of $H_{q}(Y)$ in $H_{q}(M)$ is equal to the image of $H_{q}(X)$, then $Y$ must contain some nonisovariant points of any equivariant map that is equivariantly homotopic to $f$.

Remark By construction, Browder's examples are normally cobordant to the identity; an explicit normal cobordism from the identity to $f_{0}$ is given by

$$
W=N \times[0,1] \amalg G \times S^{k} \times S^{q} \times[0,1]
$$

where $\partial_{-} W=N \times\{0\}$ and $\partial_{+} W$ is the remaining $2|G|+1$ components of the boundary, and one can obtain a normal cobordism to $f$ by adding 1 -handles equivariantly along 
the top part of the boundary. More generally, results of K H Dovermann [15] imply that one can always construct equivariant normal cobordisms to equivariant normal maps if the Gap Hypothesis holds and the map is already an equivariant homotopy equivalence on the singular set as in Browder's examples.

However, it is also possible to construct examples like Browder's that are not cobordant to highly connected maps. It suffices to let $k \equiv 0(4)$ and replace $G \times S^{k} \times S^{q}$ by $G \times S(\gamma)$, where the latter is the sphere bundle of a fiber homotopically trivial vector bundle $\gamma$ over $S^{k}$ with nontrivial rational Pontryagin classes; one must also replace the equivariant folding map from $G \times S^{k} \times S^{q}$ to $N$ by its composite with the identity on $G$ times a fiber homotopy equivalence from $S(\gamma)$ to $S^{k} \times S^{q}$. Characteristic number arguments imply the map obtained in this fashion is not cobordant to a $k$-connected map. Of course, a degree 1 map of this type does not have the bundle data required for a normal map in the sense of equivariant surgery theory.

\section{Generalizations to nonsemifree actions}

We have assumed our actions are semifree in order to keep the discussion of normal straightening as simple as possible. In fact, the main obstruction to proving analogs of Theorems 1.1, 2.1, and 2.2 for arbitrary actions is to prove a suitable generalization of the result on normal straightening. One step in carrying this out is to develop some methods for analyzing the equivariant fiber homotopy type of certain equivariant vector bundles. In the semifree case the base spaces for these bundles all have trivial $G$ actions, and we were able to study the bundles directly with a minimum of equivariant homotopy theoretic machinery. However, if the action on the ambient manifold is not semifree, then we must also consider equivariant vector bundles over the various fixed point sets of proper subgroups of $G$, and these bases will usually have nontrivial group actions. Working with such objects requires the sorts of constructions developed by Schultz $[39 ; 40]$ as well as the full force of S Waner's work on the equivariant fiber homotopy types of equivariant fiber bundles [48]. If $G=\mathbb{Z}_{p^{r}}$ where $p$ is prime, then the generalization is particularly direct, and with such results one can prove complete generalizations of Theorems 1.1, 2.1, and 2.2 for actions of such groups (assuming the Gap Hypothesis as usual).

\section{Equivariant homotopy equivalences}

In this section we shall show that an equivariant homotopy equivalence can be deformed to satisfy the conditions in parts (ii) and (iii) of Theorem 2.1 and thus must 
be equivariantly homotopic to an isovariant map, which we shall prove must be an isovariant homotopy equivalence.

Proposition 5.1 Let $f: M \rightarrow N$ be a homotopy equivalence of closed, oriented, semifree $G$-manifolds which satisfy the Gap Hypothesis such that all components of all fixed point sets are also orientable. Then $f$ is equivariantly homotopic to a map that is isovariant on a neighborhood of the fixed point set.

Proof We shall prove that $f$ satisfies the conditions in part (ii) of Theorem 2.1. Since $f$ defines a homotopy equivalence of fixed point sets, it follows immediately that for each component $M_{\alpha}$ of $M_{G}$ the restriction $f_{\alpha}$ of $f$ defines a homotopy equivalence from $M_{\alpha}$ to $N_{\alpha}$ and hence has degree \pm 1 . In order to apply part (ii) of Theorem 2.1 , we also need to verify the homotopy pullback condition on the equivariant normal bundles of the fixed point set components.

Let $\tau_{M}$ and $\tau_{N}$ be the equivariant tangent bundles of $M$ and $N$. We claim that the sphere bundles of $\tau_{M}$ and $f^{*} \tau_{N}$ are stably equivariantly fiber homotopically equivalent. The nonequivariant version of this statement is well known (cf Atiyah [4]) and the equivariant case is due to K Kawakubo [26].

Consider next the restriction of the stable equivariant fiber homotopy equivalence $S\left(\tau_{M}\right) \sim S\left(f^{*} \tau_{N}\right)$ to $M^{G}$. The classifying maps for the two equivariant fibrations go from $M^{G}$ to a space $\mathcal{B}$ such that $\pi_{*}(\mathcal{B}) \approx \pi_{*-1}^{G}$, where the latter denotes an equivariant stable homotopy group as in Segal [44]. On the other hand, by [44] we also know that $\mathcal{B}$ is homotopy equivalent to the product $B F \times B F_{G}$ where $B F$ classifies nonequivariant stable spherical fibrations and $B F_{G}$ is defined as in Becker-Schultz [7]. In terms of fibrations the projections of the classifying maps $M \rightarrow \mathcal{B}$ onto $B F$ and $B F_{G}$ correspond to taking the classifying maps of the fixed point subbundles and the orthogonal complements of the fixed point subbundles respectively. Therefore it follows that the corresponding subbundles for $\tau_{M}$ and $f^{*} \tau_{N}$ are stably equivariantly fiber homotopy equivalent. In particular, this means that $S\left(\alpha_{M}\right)$ and $S\left(\left[f^{G}\right]^{*} \alpha_{N}\right)$ are stably equivariantly fiber homotopy equivalent because they induce homotopic maps from $M^{G}$ into $B F_{G}$.

As usual, write $M^{G}$ as a disjoint union of components $M_{\alpha}$, and for each $\alpha$ let $q_{\alpha}$ be the codimension of $M_{\alpha}$. Furthermore, denote the fiber representation at a point of $M_{\alpha}$ by $V_{\alpha}$. The stabilization map $F_{G}\left(V_{\alpha}\right) \rightarrow F_{G}$ is $\left(q_{\alpha}-2\right)$-connected by the considerations of [Sc1], and the Gap Hypothesis implies that $\operatorname{dim} M_{\alpha} \leq q_{\alpha}-2$. Therefore we can destabilize the stable fiber homotopy equivalence from $S\left(\xi_{\alpha}\right)$ to $S\left(\left[f^{G}\right]^{*} \omega_{\alpha}\right)$ and obtain a genuine equivariant fiber homotopy equivalence. Choose such an equivariant fiber homotopy equivalence, say $\Phi$. It is then an elementary 
exercise to deform $f \mid \cup_{\alpha} D\left(M_{\alpha}\right)$ equivariantly relative the zero section so that one obtains the radial extension of $\Phi$ at the other end of the deformation. By the equivariant homotopy extension property one can extend this homotopy to all of $M$.

Our choice of fiber homotopy equivalences was arbitrary, but it is possible to find a canonical choice up to homotopy using equivariant $S$-duality (see Wirthmüller [51]) and Kawakubo's result; in fact, one must work with the latter to prove a uniqueness result for isovariant deformations as in [10], and we shall explain this in a subsequent article.

The preceding result and part (iii) of Theorem 2.1 reduce the proof of Theorems 1.1 and 2.2 to the following two results:

Proposition 5.2 Suppose that $f$ satisfies the conditions of the previous result, including the condition that $f$ is isovariant on a neighborhood of the fixed point set. Then $f$ is homotopic to an almost isovariant map.

Proposition 5.3 If $f$ as above is isovariant, then $f$ is an isovariant homotopy equivalence.

We shall prove these results in order.

Proof of Proposition 5.2 The first step is to construct an equivariant homotopy from $f$ to a mapping $f_{1}$ such that the homotopy is fixed on a neighborhood of the fixed point set and $f_{1}$ is smoothly equivariantly transverse to the fixed point set; there are no problems with equivariant transversality obstructions because the relevant part of the domain has a free $G$-action. By construction the transverse inverse image of the fixed point set is the set of nonisovariant points, and it splits into a union of smooth submanifolds $V_{\alpha}=f_{1}^{-1}\left(N_{\alpha}\right)$. Note that $\operatorname{dim} V_{\alpha}=\operatorname{dim} N_{\alpha}=\operatorname{dim} M_{\alpha}$ which is less than half the dimensions of $M$ and $N$.

By construction the image of $f_{1} \mid V_{\alpha}$ is contained in $N_{\alpha}$, so let $k_{\alpha}$ be the associated map from $V_{\alpha}$ to $N_{\alpha}$; if $h_{\alpha}: N_{\alpha} \rightarrow M_{\alpha}$ is determined by a homotopy inverse to $f_{1}$, then the map $h_{\alpha}{ }^{\circ} k_{\alpha}$ is equivariantly homotopic to the inclusion of $V_{\alpha}$ in $M$. By general position it follows that the latter is also equivariantly homotopic to a map into $D\left(M_{\alpha}\right)-M_{\alpha}$ and in fact can be approximated by a smooth equivariant embedding $e_{\alpha}$; in fact, the numerical condition in the Gap Hypothesis is strong enough to guarantee that $e_{\alpha}$ is equivariantly isotopic to the inclusion. Since the image of $e_{\alpha}$ is contained in a tubular neighborhood of $M_{\alpha}$, the Equivariant Isotopy Extension Theorem implies the inclusion is isotopic to a smooth equivariant embedding of $V_{\alpha}$ in a tubular neighborhood 
and hence the image of the inclusion itself must also be contained in some tubular neighborhood. Since this is true for every $\alpha$, it is also true for the entire set of nonisovariant points.

By Theorem 2.1 and the preceding propositions we know that $f$ is equivariantly homotopic to an almost isovariant mapping, and by [20] it is also equivariantly homotopic to an isovariant mapping.

Proof of Proposition 5.3 By [20, Proposition 4.1, page 27], the map $f$ is isovariantly homotopic to a map $f_{0}$ such that for each $\alpha$ we have $f\left(D\left(M_{\alpha}\right)\right) \subset D\left(N_{\alpha}\right)$, $f\left(S\left(M_{\alpha}\right)\right) \subset S\left(N_{\alpha}\right)$, and

$$
f\left(M-\cup_{\alpha} \text { Int } D\left(M_{\alpha}\right)\right) \subset N-\cup_{\alpha} \text { Int } D\left(N_{\alpha}\right) .
$$

Furthermore, using [20, Theorem 4.4, pages 29-31] one can further deform this map to some $f_{1}$ that is fiber preserving on the tubular neighborhoods and maps disk fibers to disk fibers by cones of maps over the boundary spheres (that is, the map is normally straightened in the sense of [20, page 31]). It will suffice to prove that $f_{1}$ is an isovariant homotopy equivalence, so without loss of generality we might as well assume that $f$ itself is normally straightened.

By the isovariant Whitehead Theorem established in Section 4 of [20], the map $f$ is an isovariant homotopy equivalence if $f$ defines a homotopy equivalence from $M-M^{G}$ to $N-N^{G}$. General position considerations imply that $f$ induces an isomorphism of fundamental groups, and therefore it suffices to check that $f$ defines an isomorphism in homology with twisted coefficients in the group ring of the fundamental group. Exact sequence and excision arguments show that the latter holds if $f$ induces homotopy equivalences from $M$ to $N$, from $M^{G}$ to $N^{G}$, and from $\bigsqcup S\left(\xi_{\alpha}\right)$ to $\bigsqcup S\left(\omega_{\alpha}\right)$. The first two of these follow because $f$ is an equivariant homotopy equivalence. To prove the third property first note that for each $\alpha$ the homotopy fibers of $S\left(\xi_{\alpha}\right) \subset D\left(\xi_{\alpha}\right)$ and $S\left(\omega_{\alpha}\right) \subset D\left(\omega_{\alpha}\right)$ are simply the fibers of the sphere bundles; since each $D\left(\xi_{\alpha}\right)$ maps to $D\left(\omega_{\alpha}\right)$ by a homotopy equivalence, it suffices to know that a fiber of $S\left(\xi_{\alpha}\right)$ maps to a fiber of $S\left(\omega_{\alpha}\right)$ with degree \pm 1 . This follows directly from the construction of the isovariant map; the first step was to make an equivariant homotopy equivalence normally straightened near the fixed point set, and the equivariant deformation in part (iii) of Theorem 2.1 is constant near some fiber of $S\left(\xi_{\alpha}\right)$.

\section{Recognizing isovariant homotopy equivalences}

One can combine Proposition 5.3 with Theorem 1.1 to obtain the following specialization of the Isovariant Whitehead Theorem: 
Theorem 5.4 Let $f: M \rightarrow N$ be a continuous isovariant mapping of oriented closed semifree smooth $G$-manifolds that satisfy the Gap Hypothesis. Then $f$ is an isovariant homotopy equivalence if and only if $f$ is an ordinary homotopy equivalence (forgetting the group action), and the associated map of fixed point sets $f^{G}: M^{G} \rightarrow N^{G}$ is also a homotopy equivalence.

Proof of Theorem 5.4 The necessity of the conditions is immediate, so we only need to check that the latter are also sufficient. Since smooth $G$-manifolds have the equivariant homotopy types of $G-\mathrm{CW}$ complexes (cf Illman [24]), the Equivariant Whitehead Theorem of Illman [23] and T Matumoto [29] and the hypotheses imply that $f$ must be an equivariant homotopy equivalence. Since $f$ is an isovariant map, it follows from Proposition 5.3 that $f$ is an isovariant homotopy equivalence.

Remark (Extension to nonsemifree actions) We have mentioned that Theorem 1.1 extends to at least some smooth actions that are not semifree, and in fact the same is true for Proposition 5.3. Given these, one can also extend Theorem 5.4 provided the final clause about $f^{G}$ is replaced with the following: ... and for each subgroup $H \subset G$ the associated map of $H$-fixed point sets $f^{H}: M^{H} \rightarrow N^{H}$ is also an ordinary homotopy equivalence. (Note: since the group $G$ acts trivially on its fixed point set, there is no difference between equivariant and ordinary homotopy equivalences of the singular sets - that is, the points where the action is not free — in the semifree case.)

\section{Final remarks}

Questions about the role of the Gap Hypothesis in transformation groups have been around for some time (cf Schultz [41]). Such questions rarely have clear cut answers, but the main results of this paper strongly suggest that the usefulness of the Gap Hypothesis is closely related to

(1) the strong implications of isovariance for analyzing existence and classifications questions for group actions,

(2) some very close relationships between isovariant homotopy and equivariant homotopy when the Gap Hypothesis holds.

We shall discuss a few aspects of these points in this section. 


\section{Extending Theorem 1.1 to other cases}

Since the Gap Hypothesis was used at several crucial points in the proof of our main theorems, one might reasonably expect that these results do not necessarily hold if the Gap Hypothesis fails. Despite this, there are some situations in which one can prove analogs of Theorem 1.1, particularly when $G$ is cyclic of prime order and the difference $\operatorname{dim} M-2 \operatorname{dim} M^{G}$ is equal to 1 or 0 . In particular, if we also assume that $G$ is cyclic of prime order and there is only one component with maximal dimension, then one can use surgery theory to prove generalizations of Theorem 1.1. If $G$ has order 2 and the dimension difference is zero, then this is established in [42], and the other cases will be shown in a forthcoming paper by K H Dovermann and the author. On the other hand, the previously quoted results of results of Longoni and Salvatore [27] imply that the analog of Theorem 1.1 does not necessarily hold if $G$ has order 2 and $M$ is not simply connected.

In a subsequent paper we shall use equivariant function spaces as in [38] and [7] to construct systematic families of equivariant homotopy equivalences that are not homotopic to isovariant maps in situations where the Gap Hypothesis fails. In particular, we shall construct connected examples where $G$ is cyclic of prime order, $\operatorname{dim} M=$ $2 \operatorname{dim} M^{G}$, and there are two components with the maximal dimension.

\section{Implications for equivariant surgery}

The methods and results of [20] provide a means for analyzing isovariant homotopy theory — and its relation to equivariant homotopy theory — within the standard framework of algebraic topology. Therefore Theorem 1.1 and the conclusions of [20] suggest a two step approach to analyzing smooth $G$-manifolds within a given equivariant homotopy type if the Gap Hypothesis does not necessarily hold; namely, the first step is to study the obstructions to isovariance for an equivariant homotopy equivalence and the second step is to study one of the versions of the isovariant surgery theories in [43] or [49]. This approach seems especially promising for analyzing classification questions by means of surgery theory and homotopy theory.

There are also other indications of very close ties between the validity of Theorem 1.1 and potential extensions of equivariant surgery to cases outside the Gap Hypothesis range. The extensions of Theorem 1.1 mentioned in the preceding paragraphs use results on equivariant surgery just outside the Gap Hypothesis range, and in particular they depend heavily on some corresponding extensions of results from equivariant surgery. Of course, one would also like to have more homotopy theoretic proofs for such results. On the other hand, the counterexamples to Theorem 1.1 in certain cases 
also imply that one cannot expect to have purely algebraic obstructions to equivariant surgery (based upon data like the equivariant fundamental group) in some situations that are very close to those considered by Dovermann [14] or by A Bak and M Morimoto [5; 6]. This seems to reflect the failure of the equivariant $\pi-\pi$ theorem (compare Dovermann-Rothenberg [17]) in certain cases where the Gap Hypothesis is not valid (see the last section of Chapter I in Dovermann-Schultz [18] for quite different examples along these lines).

\section{A dual approach}

The results of this paper also raise questions about a priori descriptions for isovariant homotopy types of semifree action in equivariant terms when the gap hypothesis holds. Theorem 5.4 is a simple observation in this direction. Another way of looking at the question is to consider the implications of the main theorem for a dual approach to the problem in which one begins by trying to work with the free parts of the group actions on the manifolds; suh an approach might be particularly useful if one wishes to generalize the main result to nonsmoothable actions using tools from controlled topology. If we are given two finite equivariant $\mathrm{CW}$ complexes $M$ and $N$ with semifree actions of the group $G$ that are equivariantly homotopy equivalent and their orbit spaces are given by $M^{*}$ and $N^{*}$ respectively, then purely formal considerations imply that the one point compactifications of $M^{*}-M^{G}$ and $N^{*}-N^{G}$ are homotopy equivalent because these spaces are homeomorphic to the quotient spaces $M^{*} / M^{G}$ and $N^{*} / N^{G}$ respectively. If $M$ and $N$ are smooth manifolds and the Gap Hypothesis holds, then Theorem 1.1 implies that this canonical homotopy equivalence of one point compactifications comes from a proper homotopy equivalence between $M^{*}-M^{G}$ and $N^{*}-N^{G}$ and this mapping can be compactified in the sense of Quinn's work on ends of maps [36]. A good understanding of this from the viewpoint of controlled topology as in [36] would be extremely useful for studying possible extensions of Theorem 1.1 to group actions that are not necessarily smooth but are still somehow well behaved topologically. 


\section{References}

[1] J F Adams, On the groups $J(X), I$, Topology 2 (1963) 181-195 MR0159336

[2] A Assadi, W Browder, On the existence and classification of extensions of actions on submanifolds of disks and spheres, Trans. Amer. Math. Soc. 291 (1985) 487-502 MR800249

[3] A H Assadi, P Vogel, Actions of finite groups on compact manifolds, Topology 26 (1987) 239-263 MR895576

[4] MF Atiyah, Thom complexes, Proc. London Math. Soc. (3) 11 (1961) 291-310 MR0131880

[5] A Bak, M Morimoto, Equivariant surgery and applications, from: "Topology Hawaii (Honolulu, HI, 1990)”, World Sci. Publishing, River Edge, NJ (1992) 13-25 MR1181478

[6] A Bak, M Morimoto, Equivariant surgery with middle-dimensional singular sets I, Forum Math. 8 (1996) 267-302 MR1387697

[7] J C Becker, R E Schultz, Equivariant function spaces and stable homotopy theory I, Comment. Math. Helv. 49 (1974) 1-34 MR0339232

[8] A Beshears, G-isovariant structure sets and stratified structure sets, PhD thesis, Vanderbilt University (1997)

[9] W Browder, Surgery and the theory of differentiable transformation groups, from: "Proc. Conf. on Transformation Groups (New Orleans, La., 1967)", Springer, New York (1968) 1-46 MR0261629

[10] W Browder, Isovariant homotopy equivalence, Abstracts Amer. Math. Soc. 8 (1987) 237-238

[11] W Browder, F Quinn, A surgery theory for G-manifolds and stratified sets, from: "Manifolds-Tokyo 1973 (Proc. Internat. Conf., Tokyo, 1973)", Univ. Tokyo Press, Tokyo (1975) 27-36 MR0375348

[12] S Cappell, S Weinberger, Surgery theoretic methods in group actions, from: "Surveys on surgery theory, Vol. 2", Ann. of Math. Stud. 149, Princeton Univ. Press, Princeton, NJ (2001) 285-317 MR1818776

[13] A Dold, Partitions of unity in the theory of fibrations, Ann. of Math. (2) 78 (1963) 223-255 MR0155330

[14] KH Dovermann, $\mathbb{Z}_{2}$ surgery theory, Michigan Math. J. 28 (1981) 267-287 MR629360

[15] K H Dovermann, Almost isovariant normal maps, Amer. J. Math. 111 (1989) 851-904 MR1026286

[16] K H Dovermann, T Petrie, G surgery II, Mem. Amer. Math. Soc. 37 (1982) xxiii+118 MR652862 
[17] K H Dovermann, M Rothenberg, Equivariant surgery and classifications of finite group actions on manifolds, Mem. Amer. Math. Soc. 71 (1988) viii+117 MR920965

[18] K H Dovermann, R Schultz, Equivariant surgery theories and their periodicity properties, Lecture Notes in Mathematics 1443, Springer, Berlin (1990) MR1077825

[19] E Dror Farjoun, Homotopy and homology of diagrams of spaces, from: "Algebraic topology (Seattle, Wash., 1985)", Lecture Notes in Math. 1286, Springer, Berlin (1987) 93-134 MR922924

[20] G Dula, R Schultz, Diagram cohomology and isovariant homotopy theory, Mem. Amer. Math. Soc. 110 (1994) viii+82 MR1209409

[21] B Gray, Homotopy theory, Academic Press [Harcourt Brace Jovanovich Publishers], New York (1975) MR0402714

[22] B Hughes, S Weinberger, Surgery and stratified spaces, from: "Surveys on surgery theory, Vol. 2", Ann. of Math. Stud. 149, Princeton Univ. Press, Princeton, NJ (2001) 319-352 MR1818777

[23] S Illman, Equivariant algebraic topology, Ann. Inst. Fourier (Grenoble) 23 (1973) 87-91 MR0358761

[24] S Illman, Smooth equivariant triangulations of $G$-manifolds for $G$ a finite group, Math. Ann. 233 (1978) 199-220 MR0500993

[25] L Jones, Combinatorial symmetries of the m-dimensional ball, Mem. Amer. Math. Soc. 62 (1986) iv+124 MR849939

[26] K Kawakubo, Compact Lie group actions and fiber homotopy type, J. Math. Soc. Japan 33 (1981) 295-321 MR607946

[27] R Longoni, P Salvatore, Configuration spaces are not homotopy invariant, Topology 44 (2005) 375-380 MR2114713

[28] W Lück, I Madsen, Equivariant L-theory I, Math. Z. 203 (1990) 503-526 MR1038715

[29] T Matumoto, On G-CW complexes and a theorem of JHC Whitehead, J. Fac. Sci. Univ. Tokyo Sect. IA Math. 18 (1971) 363-374 MR0345103

[30] R J Milgram, P Löffler, The structure of deleted symmetric products, from: "Braids (Santa Cruz, CA, 1986)", Contemp. Math. 78, Amer. Math. Soc., Providence, RI (1988) 415-424 MR975092

[31] J Milnor, Lectures on the h-cobordism theorem, Notes by L. Siebenmann and J. Sondow, Princeton University Press, Princeton, N.J. (1965) MR0190942

[32] R S Palais, The classification of G-spaces, Mem. Amer. Math. Soc. No. 36 (1960) MR0177401 
[33] T Petrie, Pseudoequivalences of $G$-manifolds, from: "Algebraic and geometric topology (Proc. Sympos. Pure Math., Stanford Univ., Stanford, Calif., 1976), Part 1", Proc. Sympos. Pure Math., XXXII, Amer. Math. Soc., Providence, R.I. (1978) 169-210 MR520505

[34] T Petrie, One fixed point actions on spheres I, II, Adv. in Math. 46 (1982) 3-14, 15-70 MR676986

[35] A Phillips, Submersions of open manifolds, Topology 6 (1967) 171-206 MR0208611

[36] F Quinn, Ends of maps II, Invent. Math. 68 (1982) 353-424 MR669423

[37] M Rothenberg, Review of the book "Equivariant Surgery Theories and Their Periodicity Properties” by K H Dovermann and R Schultz, Bull. Amer. Math. Soc. (2) 28 (1993) 375-381

[38] R Schultz, Homotopy decompositions of equivariant function spaces I, Math. Z. 131 (1973) 49-75 MR0407866

[39] R Schultz, Spherelike G-manifolds with exotic equivariant tangent bundles, from: "Studies in algebraic topology", Adv. in Math. Suppl. Stud. 5, Academic Press, New York (1979) 1-38 MR527243

[40] R Schultz, Differentiable group actions on homotopy spheres II: Ultrasemifree actions, Trans. Amer. Math. Soc. 268 (1981) 255-297 MR632531

[41] R Schultz, Problems submitted to the AMS summer research conference on group actions, from: "Group actions on manifolds (Boulder, Colo., 1983)", Contemp. Math. 36, Amer. Math. Soc., Providence, RI (1985) 513-568 MR780979

[42] R Schultz, A splitting theorem for manifolds with involution and two applications, J. London Math. Soc. (2) 39 (1989) 183-192 MR989929

[43] R Schultz, Isovariant homotopy theory and differentiable group actions, from: "Algebra and topology 1992 (Taejŏn)”, Korea Adv. Inst. Sci. Tech., Taejŏn (1992) 81-148 MR1212981

[44] G B Segal, Equivariant stable homotopy theory, from: "Actes du Congrès International des Mathématiciens (Nice, 1970), Tome 2", Gauthier-Villars, Paris (1971) 59-63 MR0423340

[45] S H Straus, Equivariant codimension one surgery, $\mathrm{PhD}$ thesis, University of California, Berkeley (1972)

[46] T tom Dieck, Transformation groups and representation theory, Lecture Notes in Mathematics 766, Springer, Berlin (1979) MR551743

[47] C T C Wall, Surgery on compact manifolds, Mathematical Surveys and Monographs 69, American Mathematical Society, Providence, RI (1999) MR1687388

[48] S Waner, Equivariant classifying spaces and fibrations, Trans. Amer. Math. Soc. 258 (1980) 385-405 MR558180 
[49] S Weinberger, The topological classification of stratified spaces, Chicago Lectures in Mathematics, University of Chicago Press, Chicago, IL (1994) MR1308714

[50] S Weinberger, Higher $\rho$-invariants, from: “Tel Aviv Topology Conference: Rothenberg Festschrift (1998)”, Contemp. Math. 231, Amer. Math. Soc., Providence, RI (1999) 315-320 MR1707352

[51] K Wirthmüller, Equivariant S-duality, Arch. Math. (Basel) 26 (1975) 427-431 MR0375297

Department of Mathematics, University of California at Riverside Riverside CA 92521, USA

schultz@math.ucr.edu

Received: 29 September 2005 Revised: 8 May 2006 